\title{
Intelligent vehicular maintenance system using IoT, Mobile application, internet- enabled vehicles and vehicular could
}

\author{
Noor Ghusen Al-Jaafari \\ Imran Ahmed \\ Anjum Zameer Bhatt \\ Mohammed Samiulla Khan
}

\author{
Middle East College \\ Middle East College \\ Middle East College \\ Middle East College
}

With the rapid increase of autonomous vehicles, safe and secure use and vehicle maintenance have become a concern for some vehicle owners. Safe and secure use of vehicles and maintenance is always essential, the current systems lack safety and security in autonomous vehicles. This paper is aimed at suggesting an autonomous maintenance system via IoT and mobile technology to indorse the safer and secure use and maintenance of autonomous vehicles. The development of cloud computing, internet-of-things, wireless sensor networks and so forth, has opened a new horizon for solving problems associated with the safer and secure use of the autonomous vehicles and their maintenance. This paper presents intelligent IoT enabled mobile application for the safer and secure use of autonomous vehicles along with the maintenance, the paper also discusses how the data from this system can be used in the vehicular cloud and support in autonomous internet-connected vehicles

\section{Introduction:}

The evolution of the Internet of Things over the past years sweeps all over the world and various electronic devices, has become very important and deserves to be given some attention. The Internet of Things enables to show the jewel of the future vision in the world of technology and digital as it was able to understand the functions and hear and think about them and make them in the appropriate format according to the functions assigned to the device and it succeeded in making the devices capable of speaking with each other, and can synchronize data Easily and quickly and facilitate the exchange of information. The Internet of Things has been able to transform devices or objects from traditional to smart devices and high-speed through the using of basic technologies in the production of the Internet of things and the most prominent of these technologies (computing, communications technologies, sensor networks, applications, etc. (. In this paper we will discuss the topic of converting vehicles from ordinary to smart. Through previous studies in this area and experimental procedures have been concluded that the knee can communicate with its owner via mobile phone, as this type of technology makes it easier for the owner of the vehicle to understand what is going on in the vehicle through the system in them, instead of showing logos on the screen The vehicle only sends logos on the mobile phone, for example, in the case that the owner of the vehicle closed the vehicle and forgot the lamps of the vehicle is open The system sends to the owner of the vehicle on the mobile phone logo stating that the vehicle lamps are still open. This system is also designed to provide the safety, security and welfare of the vehicle and its owner.

\section{Develop mobile applications and connect them to the vehicle:}

As we know today, the vehicle industry is growing and is becoming connected to smart screens 


\section{Journal of Student Research}

Fourth Middle East College Student Research Conference, Muscat, Sultanate of Oman

Fig1 that allow you to use it similar to the use of mobile phones and it supports voice and video calling and also text messaging and opening applications such as YouTube and WhatsApp and others. It also allows Bluetooth and Internet connectivity by analyzing signals from the cloud. It also shows the signals coming from the sensors that connected to the vehicle into the vehicle screen after being analyzed in the Embedded system. Whereas, the Embedded system is specialized in analyzing signals from sensors, Internet and Bluetooth signals.

From here we propose the idea of creating an application for the mobile phone connected to the vehicle, as after the establishment of the application is loaded in the screen of the vehicle as it is like other applications that depend on showing the location or signals in the vehicle, As this application is dedicated to vehicle alerts and maintenance requests and by downloading it in the vehicle. This application works by receiving the signal from the vehicle control system after the analysis of the signal in the Embedded system, where the signal appears on the vehicle screen and at the same time is sent to the application in the mobile phone as notification. Also, one of the features of this system is the possibility of requesting a vehicle maintenance service in time of need by determining the location using Google Map. For example, in the case of a brake failure, a signal from the brake is sent to the control system and analyzes the signal using the Embedded system. After that a (illuminated signal) is sent to the car screen and at the same time by using Bluetooth or the Internet is sent to the mobile phone application as notification to inform the owner of the vehicle that the parking brake is disabled, With a feature in the application that allows maintenance request, through that it which allows the owner of the vehicle can determine the location of the vehicle and show maintenance workshops near the site and then the maintenance request is made after the selection of one of the maintenance workshops to transport the vehicle to the maintenance workshop and repair or be repaired in the same location and thus the task was accomplished in a short time and less effort.

\section{Embedded System:}

Embedded means something that is related to something else, and a built-in system is a system specifically designed to perform a specific task or a number of specific tasks, i.e. it is designed for specific purposes, a controller or processor based on a system that is designed to perform a specific task or several specific tasks. The built-in system can be considered a hardware computer with software. A built-in system can be a stand-alone system or part of another large system, i.e. built into a larger product so it can be invisible to the user. The vehicle control is not without the Embedded chip, Fig2 It is responsible for receiving signals from sensitive devices and other vehicle parts such as lighting, gasoline tank and tire. They are attached to the sensors in all parts of the vehicle. By receiving signals, it analyzes that signal and shows it on the vehicle screen for example, when the car needs gasoline, the sensors are mounted by sending a signal to the Embedded system in the vehicle control. The Embedded system analyzes the gasoline shortage signal after which the vehicle appears illuminated in the form of filling gasoline, Fig3 (as we all know that this indication indicates that the vehicle needs gasoline).

Figure 1. diagram for how Embedded system works

\section{Discussion:}

The world is evolving at an ever-increasing pace. Today, the key to evolution is technology, and software is the pillar of technology that can never be overcome.

While programming and technology are involved in all the details of our daily lives, they have left no room without having the upper hand in it. Year after year, the proportion of vehicles with the most modern and sophisticated technologies is increasing and the proportion of technological 
components in them increases.

1. Saving time and effort:

2. Enhance communication between customers and companies.

3. Provide better security and safety.

Through the presence of sensors in all parts of the vehicle in small sizes or varying size and the presence of the control system in the vehicle, where the vehicle is damaged or a traffic accident or even the arrival of an annual maintenance date of the vehicle, a signal is sent through the sensors to the vehicle control system and this signal is sent using wireless network or Bluetooth via mobile phone as Fig4 shows, through the presence of a program dedicated to maintenance services Through this program allows to call and send messages or pressure on the options displayed in the emergency program to request service maintenance of the vehicle and also allows the feature of automatic identification of the location of the vehicle in Google Map.

With a wireless communication point between the vehicle and the mobile phone of the owner of the vehicle through the Application dedicated to request maintenance services, through which the customer can follow the latest vehicle maintenance events or request maintenance service from maintenance workshops and companies.

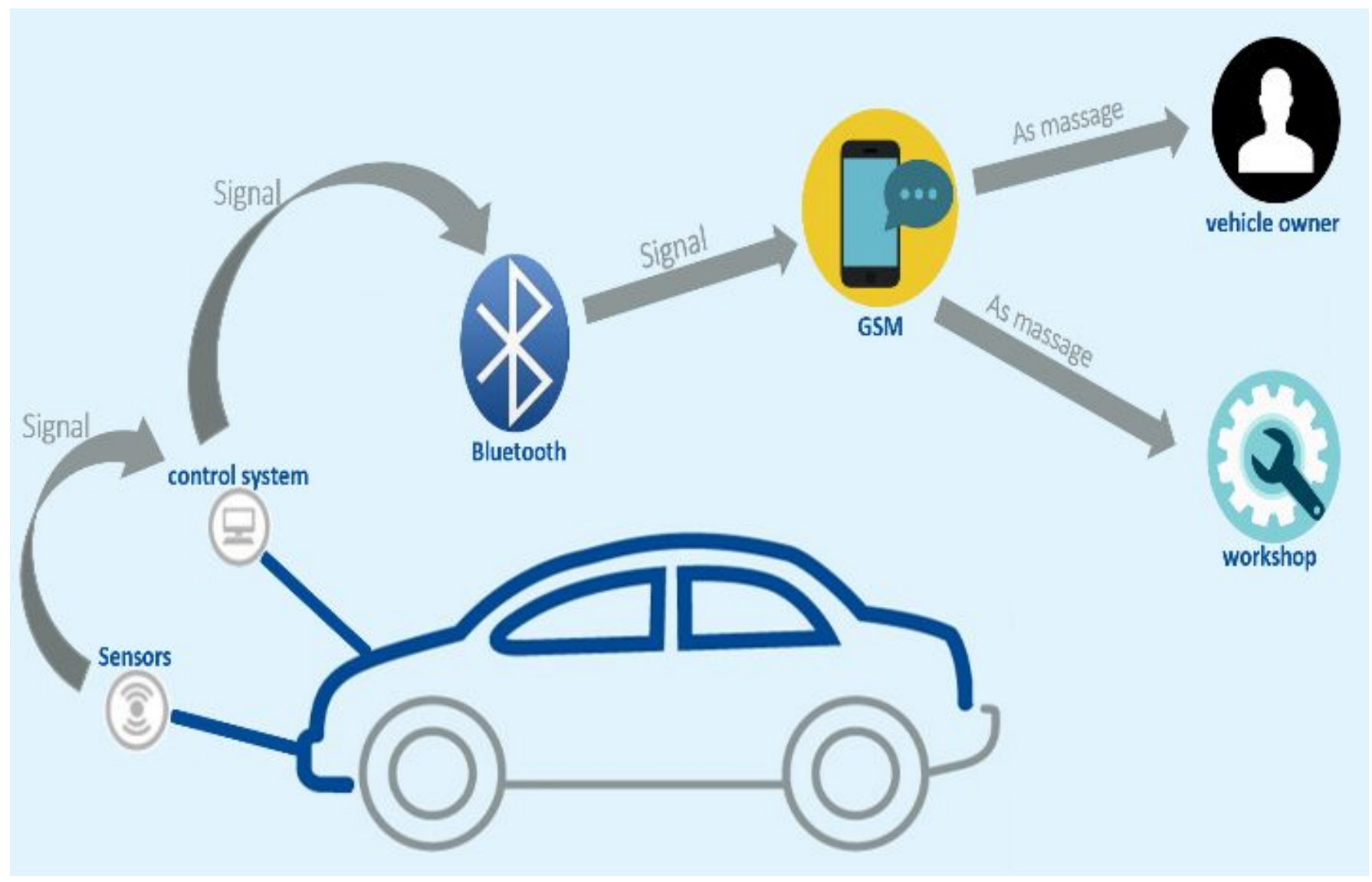

Figure 2. Steps how the system work

\section{Results:}

Nowadays, technological advances surround you from all directions, where you feel wherever you go, whether you're at home, on the street, at work or even inside your own vehicle. Speaking of your own vehicle, many people used to rely solely on their vehicles as their only means of transportation, forcing some to spend most of their time traveling from one place to another, until 
they thought the vehicle was their second home.

Since we live in a modern and sophisticated era, where comfort and safety are the most important for humans, we may give some attention in the aspect of vehicle maintenance by focusing on transforming vehicles from a means of mobility to a means of comfort and safety

As the technology enters into the maintenance of vehicles or request maintenance services, it is interested in improving the performance of the car in terms of comfort, speed and safety.

Table 1 shows a simple comparison showing the changes that occur after and before entering the system. Through the presence of this system that allows you to know the malfunction of the vehicle by sending a signal through the vehicle control system or the owner of the vehicle can request Other services such as transporting or washing the vehicle, etc. The most prominent of these positive results after the introduction of technology or the Internet of things in the system of maintenance and safety of the vehicle is.

- Saving time and effort: There is no doubt that the most important benefits of this system is to save the time required to perform tasks, it was the process of moving from one place to another to search for a maintenance workshop takes a lot of time and effort and some of us may have difficulty going to the maintenance workshops while the presence of this The system does not need to search and go to maintenance workshops no matter how close or far away from us.

- Enhance communication between customers and companies: Providing this system gives the correct way to communicate between customers and maintenance workshops or companies to know or request repair of the fault that occurs in the vehicle and all that makes it easier for customers to know the latest maintenance coordinates of their vehicles through the application dedicated mobile phones to request maintenance services available without the need to go workshops maintenance And fill in the papers or stand in the waiting rooms and also makes it easier for maintenance workshops or companies to send the latest vehicle data and also offers if available to the customer's phone directly, and when inquiries can send text conversations directly via chat feature in the application. All this enhances communication between the customer and the employees in maintenance workshops or companies.

The following Fig5 shows the first model of the application.

Figure 3. Model of the application

- Provide better security and safety: This system can provide security and safety for the vehicle as this system works to send the vehicle to the mobile phone and indicate the type of malfunction that occurred in the vehicle where the owner can know the malfunction and its place in the body of the vehicle and therefore the customer can repair the malfunction before a malfunction occurs Larger.

\section{Conclusion:}

Today we believe that technology has become a part of our daily lives and it is difficult to do without it. It also has many useful aspects that help us get things done easily whether it is via mobile phone or various technological devices. Through this research, the idea of helping the community overcome some of the problems faced by customers and maintenance workshops to create an integrated digital environment and free from difficulties in accomplishing life tasks, and the presence of this system is a positive point in the digital side as all segments of society do not dispense with different Systems and applications, whether on their mobile phones or in various 


\section{Journal of Student Research}

Fourth Middle East College Student Research Conference, Muscat, Sultanate of Oman

electronic devices and through which they are accomplishing their tasks, the system is characterized by simplicity in design and ease of use It is available to users in their mobile phones only, which facilitates the process more communication between customers and owners of Maintenance workshop.

\section{Acknowledgement:}

I sincerely thank ALLAH for giving me this precious opportunity, and I would also like to thank MEC for providing various resources and support to me. Special thanks were given to Supervisor Imran Ahmed for guiding me in all the steps to complete this project. Finally, I would like to say thank you to everyone who provided me with support and advice in the completion of this project.

Figure 4. Smart Screen for vehicle

Figure 5. Embedded chip

Figure 6. comparison between after and before entering the system

\section{References:}

1. (2015)., Simon Ninan19) August 2015) The IoT-connected car of today-and tomorrow.

2. Vitaly Petrov.,( February 2017) "Vehicle-Based Relay Assistance for Opportunistic Crowdsensing overNarrowband IoT (NB-IoT)".

3. GSMA Head (July 2014) "Understanding the Internet of Things (IoT)" .

4. Mario G. Beruvides, James Simonton (April 2015 )" Public Transportation Vehicle Maintenance".

5. Author links open overlay panelC.M.Monteiro (2016.11.012) "Vehicles Emerging Technologies from Maintenance Perspective".

6. Paul Ryabchuk (September 13, 2018) "How Can the Automotive Industry Use Internet of Things (IoT) Technology?".

7. Paul Green (September 2013) "Review Article Automotive Technology and Human Factors Reiser 